$\xi=-$ 㕄

\title{
Geomechanical evaluation of reservoirs in the coastal swamp, Niger delta region of Nigeria
}

\author{
Thomas Akpan Harry ${ }^{1}$, Sunday Edet Etuk ${ }^{2}$, Inyang Namdie Joseph ${ }^{2}$, Okoli Emeka Austin ${ }^{3}$ \\ ${ }^{1}$ Department of Geology, Akwa Ibom State University, Mkpat Enin, Nigeria \\ ${ }^{2}$ Department of Physics, University of Uyo, Nigeria \\ ${ }^{3}$ Department of Geology, Federal University of Technology, Owerri \\ *Corresponding author E-mail: tharry.tom@gmail.com
}

\begin{abstract}
Evaluation of elastic parameters of reservoirs can be used in geomechanical modelling, wellbore stability analysis, and sanding, which can be applied in practical situation to optimize drilling, completion and productions of wells. Petrophysical analysis was done to identify various reservoirs in two wells, using sonic, neutron, gamma, resistivity and density logs. Porosity, Lithology and Water/Hydrocarbon saturation were determined. The lithology are mostly sand, shale and sandstone with sand/sandstone been the main lithology found in the reservoirs. Porosities in the two wells decreases with depth except in few cases, due to over pressured zones, caused by fluid contents. The reservoirs identified in the two wells are of economic importance due to their net pay zone ranging from $7.16 \mathrm{~m}$ to $225.25 \mathrm{~m}$, with $1067 \mathrm{~m}$ to $3507 \mathrm{~m}$ depth, which is within the Agbada formation, having a minimum an average hydrocarbon saturation of 50\%. Elastic parameters evaluated are $V_{s}, V_{p}, V_{p} / V_{s}$, Poisson ratio, Shear Impedance, Acoustic Impedance, Bulk Modulus, Shear Modulus and Young Modulus. $\mathrm{V}_{\mathrm{p}} / \mathrm{V}_{\mathrm{s}}$ and Poisson ratio was used to also infer and confirm the lithology gotten from gamma log and also used to discriminate between, oil sand, gas sand and brine sand. The Elastic properties of the reservoirs that are found mostly in the sandstone lithology varies between 15437.91 to $31522.28 \mathrm{psi}, 7.10$ to $24.78 \mathrm{Kbar}$, 5929.51 to $22339.26 \mathrm{psi}, 1.94$ to $18.06 \mathrm{Kbar}, 1.70$ to $2.31,5.34$ to $43.59 \mathrm{psi}$ of Acoustic Impedance, Bulk Modulus, Shear Impedance, Shear Modulus, Velocity ratio and Young Modulus respectively.
\end{abstract}

Keywords: Acoustic Impedance; Bulk Modulus; Shear Impedance; Shear Modulus; Young Modulus.

\section{Introduction}

The basic objective of well logging is to transform well log data into a quantitative rock property, descriptive of the reservoir (Pendrel, 2006, Agbasi et al 2013.) The ability to estimate acoustic impedance and a parameter related to shear impedance increases the interpreter's ability to discriminate between different lithologies and fluid phases (Duffaut, et al., 2000, Agbasi et al 2018), resulting in a detailed reservoir characterization for improved hydrocarbon recovery (Bassey et al, 2013). This is an improvement over conventional seismic interpretation which relies on the seismic data alone to map geological structures and identify potential exploration targets.

Angle-limited post stack volumes provide a simple way to examine the variations of seismic amplitudes with angle of incidence. The stacks are often generated into near, mid or far volumes corresponding to angles of incidence, such that amplitude variations in these volumes in target zones are mainly indicative of the reservoir fluid. The amplitudes of near angle stacks relate to changes in acoustic impedance (Connolly, 1999, Fidelis and Harry, 2014) which can be well correlated with lithologic changes, and to invert near angle stack for acoustic impedance, acoustic impedance (AI) is derived directly from the compressional sonic and density logs to provide input for the inversion algorithm. This is the conventional post stack inversion method and it has proven successful in many rock property prediction studies (Inichinbia, et al., 2014, Keumsuk, et a ., 2013. Anderson, 2009.
Wagner, et al., 2012, Okechukwu et al 2018). In some cases, acoustic impedance alone may fail or not be enough to quantify reservoir rock properties such as lithology and pore fluid, for a detailed understanding of the reservoir. An additional parameterization related to the rock physics must therefore be utilized to fully characterize the reservoir. Elastic impedance may provide the additional parameterization for increasing the quality of the reservoir characterization in such cases. This is essentially the case with the present study.

Using a linearized version of the Zeoppritz equations, derived a generalization of acoustic impedance for variable angles of incidence. This is known as elastic impedance (EI), and provides the framework to calibrate and invert high-angle seismic without reference to near offsets (angles). Elastic impedance (EI) as a function of $\mathrm{P}$-wave angle of incidence, $\theta$, is given by:

$E l=V_{p}^{(1+\sin 2 \Theta)} V_{s}^{(-8 K \sin 2 \Theta)} \rho_{b}^{(1-4 K \sin 2 \Theta)}$

Where $\mathrm{V}_{\mathrm{P}}, \mathrm{V}_{\mathrm{S}}, \rho$ and $\theta$ are $\mathrm{P}$-wave velocity, $\mathrm{S}$-wave velocity, bulk density and $\mathrm{P}$-wave angle of incidence, respectively, and $\mathrm{k}$ is a factor that is assumed to be constant and usually set to be equal to the average value of $\left(\mathrm{V}_{\mathrm{s}} / \mathrm{V}_{\mathrm{p}}\right)^{2}$ over the log interval of interest. One problem associated with elastic impedance is that it has strange dimensions (units) and the values do not scale correctly for different angles (Whitcombe 2002) as a result of raising velocity and density terms to increasing powers; the dimensions (units) vary with angle of incidence. To remove the dimensionality 
as a function of $\mathrm{P}$-wave angle of incidence and provide elastic impedance with the same dimensionality, (Whitcombe 2002) introduced the normalization constants $\mathrm{V}_{\mathrm{p}} 0, \mathrm{~V}_{\mathrm{S}} 0$ and $\rho_{0}$, and normalized the elastic impedance.

\section{Geology of Niger delta}

The Cenozoic Niger Delta is situated at the intersection of the Benue Trough and the South Atlantic Ocean where a triple junction developed during the separation of the continents of South America and Africa in the late Jurassic. Subsidence of the African continental margin and cooling of the newly created oceanic lithosphere followed this separation in early Cretaceous times. Marine sedimentation took place in the Benue Trough and the Anambra Basin formed-Cretaceous onwards. The Niger Delta started to evolve in early Tertiary times when clastic river in put increased (Udo, et al 2015, Orife and Avbovbo 2013).Generally the delta prograded over the subsidizing continental-oceanic lithospheric transition zone, and during the Oligocene spread on to oceanic crust of the Gulf of Guinea. The weathering flanks of out-cropping continental basement sourced the sediments through the Benue-Niger drainage basin. The delta has since Paleocene time's prograded a distance of more than $250 \mathrm{~km}$ from the Benin and Calabar flanks to the present delta front (Ubong et al 2017). Thickness of sediments in the Niger Delta averages $12 \mathrm{~km}$ covering a total area of about $140,000 \mathrm{~km} 2$. The Stratigraphic sequence of the Niger Delta comprises three broad lithostratigraphic units namely, (1) a continental shallow massive sand sequence the Benin Formation, (2) a coastal marine sequence of alternating sands and shales the Agbada Formation and (3) a basal marine shale unit-the Akata Formation.

The Akata Formation consists of clays and shales with minors and intercalations. The sediments were deposited in pro delta environments. The sand percentage here is generally less than $30 \%$. The Agbada Formation consists of alternating sand and shales representing sediments of the transitional environment comprising the lower delta plain (mangrove swamps, floodplain, and marsh) and the coastal barrier and fluviomarine realms. The sand percentage within the Agbada Formation varies from 30 to $70 \%$, which results from the large number of depositional off lap cycles. A complete cycle generally consists of thin fossil ferrous transgressive marine sand, followed by an off lap sequence which commences with marine shale and continues with laminated fluviomarine sediments followed by barrier sand/or fluviatile sediments terminated by another transgression (Akpankpo et al 2015; Inyang et al 2017; Okoli et al 2018). The Benin Formation is characterized by high sand percentage $(70-100 \%)$ and forms the top player of the Niger Delta depositional sequence. The massive sands were deposited in continental environment comprising the fluvial realms (braided and meandering systems) of the upper delta plain.

\section{Methodology}

The application of a force to an object is known as loading. Materials can be subjected to many different loading scenarios and a material's performance is dependent on the loading conditions. There are five fundamental loading conditions; tension, compression, bending, shear, and torsion. Tension is the type of loading in which the two sections of material on either side of a plane tend to be pulled apart or elongated. Compression is the reverse of tensile loading and involves pressing the material together. Loading by bending involves applying a load in a manner that causes a material to curve and results in compressing the material on one side and stretching it on the other. Shear involves applying a load parallel to a plane which caused the material on one side of the plane to want to slide across the material on the other side of the plane. Torsion is the application of a force that causes twisting in a material.
If a material is subjected to a constant force, it is called static loading. If the loading of the material is not constant but instead fluctuates, it is called dynamic or cyclic loading. The way a material is loaded greatly affects its mechanical properties and largely determines how, or if, a component will fail; and whether it will show warning signs before failure actually occurs. Stress is defined as the force per unit area of a material.

$$
\sigma=\frac{F}{A}
$$

$\sigma=$ Stress

$\mathrm{F}=$ Force

$\mathrm{A}=$ Area

Is the fractional deformation produced in a solid body when it is subjected to a load. Or is the ratio of the change in length to the initial length

$\varepsilon=\frac{\Delta L}{L}$

Strain is the response of a system to an applied stress. When a material is loaded with a force, it produces a stress, which then causes a material to deform. Engineering strain is defined as the amount of deformation in the direction of the applied force divided by the initial length of the material. This results in a unitless number, although it is often left in the unsimplified form, such as inches per inch or meters per meter. For example, the strain in a bar that is being stretched in tension is the amount of elongation or change in length divided by its original length. As in the case of stress, the strain distribution may or may not be uniform in a complex structural element, depending on the nature of the loading condition.

Young's modulus is the ratio of the longitudinal stress to the longitudinal strain when a solid body is loaded by longitudinal stress within the elastic limit.

This is because stress is proportional to strain. The gradient of the straight-line graph is the Young's modulus, E

$E=\frac{\operatorname{Stress}(\sigma)}{\operatorname{Strain}(\varepsilon)}$

Poisson's ratio is the ratio of lateral strain to axial strain, that is:

$\mu=\frac{\text { Lateral Strain }}{\text { Longitudinal strain }} \quad 0 \leq \mu \leq 0.5$

Shear modulus is the ratio of shear stress to shear strain.

$\mathrm{G}=\frac{\text { Snear Stress }}{\text { Shear Strair }}$

On the other hand, Bulk modulus is the ratio of the applied stress to the volumetric strain when a solid body is subjected to uniform stress throughout its surface, that is:

$$
K=\frac{E}{3(1-2 \mu)}
$$

The geo-mechanical properties can be modeled based on well logging tools such as density and acoustic velocities, Gamma Ray, Neutron. Wireline measurements were converted to mechanical properties using the equations for homogeneous isotropic and elastic rock as follows:

Poisson's Ratio

For homogeneous isotropic and elastic rock, physical rock, Poisson's Ratio is given as: 


$$
\mu=0.5\left(\frac{\left(\frac{\Delta t_{s}}{\Delta t_{c}}\right)^{2}-2}{\left(\frac{\Delta t_{s}}{\Delta t_{c}}\right)^{2}-1}\right)
$$

\section{Shear Modulus:}

For homogeneous isotropic and elastic rock, physical rock, Poisson's Ratio is given as:

$$
G(p s i)=1.34 \times 10^{10} \frac{\rho_{B}}{\Delta t_{s}}
$$

Young's Modulus:

For homogeneous isotropic and elastic rock, physical rock, Poisson's Ratio is given as:

$$
E(p s i)=1.34 \times 10^{10}\left(\frac{\rho_{B} V_{s}^{2}\left(3 V_{c}^{2}-4 V_{s}^{2}\right)}{V_{c}^{2}-V_{s}^{2}}\right)
$$

Bulks Modulus:

For homogeneous isotropic and elastic rock, physical rock, Poisson's Ratio is given as:

$$
K_{B}(p s i)=\frac{1}{C_{b}}=1.34 \times 10^{10} \rho_{B}\left(\frac{1}{\Delta t_{c}^{2}}-\frac{4}{3 \Delta t_{s}^{2}}\right)
$$

Where:-

$\mathrm{Vs}=$ Travel time of shear $(\mathrm{msec} / \mathrm{ft})$

$\mathrm{Vc}=$ Travel time of compressive $(\mathrm{msec} / \mathrm{ft})$

$\mathrm{P}_{\mathrm{b}}=$ Bulk Density

For unconsolidated sandstone, estimation of the dynamic rock mechanics properties is challenging as it is difficult to measure the shear wave in this type of formation, then alternative approach is necessary calculate these parameters.
Table 1: Constants for Simple Exponential Curve Fits To Porosity - Mechanical Properties Relationships for Sandstone and Carbonates

\begin{tabular}{lllll}
\hline $\begin{array}{l}\text { Property } \\
\text { (units) }\end{array}$ & Rock & \multicolumn{2}{l}{ Constants } & Correlation Coeffi- \\
\hline \multirow{2}{*}{$\sigma \mathrm{UCS}(\mathrm{MPa})$} & Type & A & B & cient \\
\hline \multirow{2}{*}{$\mathrm{E}_{\text {Static }}(\mathrm{GPa})$} & Carbonate & 208.08 & 0.074 & 0.71 \\
& Sandstone & 56.4 & 0.112 & 0.94 \\
$\mathrm{E}_{\text {Dynamic }}(\mathrm{GPa})$ & Carbonate & 69.05 & 0.06 & 0.87 \\
& Sandstone & 55.39 & 0.146 & 0.96 \\
& Carbonate & 66.98 & 0.042 & \\
\hline
\end{tabular}

Where: $\mathrm{P}_{\mathrm{c}}, \mathrm{P}_{\mathrm{w}}, \mathrm{P}_{\mathrm{e}}$ are the critical pressure, working bottom hole pressure and reservoir pressure respectively; $r_{e}, r_{w}$ and $R_{s}$ are the reservoir, wellbore and sanding radius respectively.

Cooke and Schneider 1983 establish general $\mathrm{V}_{\mathrm{p}} / \mathrm{V}_{\mathrm{s}}$ relationships for elastic silicate rocks by comparing in-situ and laboratory data with theoretical model data. Available velocity information was examined for data from water-saturated mud rocks and sandstones. They examine laboratory data from dry sandstone and compare with simple sphere pack and cracked media theoretical model data. Data from water-saturated rocks were similarly investigated. The results of the relationships established between $V_{p}$ and $V_{s}$, are then applied to calculations of rock dynamic moduli. Finally, the general $\mathrm{V}_{\mathrm{p}^{-}} \mathrm{V}_{\mathrm{s}}$ trends versus depth were estimated for Gulf Coast elastics.

\section{Results and discussion}

Petrophysical and Elastic analysis was carried out for all the identified hydrocarbon intervals, from the two wells studied in the Niger Delta Fields using suites of geophysical well logs. This research found that the bulk of the hydrocarbon encountered in the Niger Delta basin was found to be within a depth range of 3,500.5 $11,507 \mathrm{ft}(1,067 \mathrm{~m}-3,507 \mathrm{~m})$ as compared to the values gotten by Agbasi et al 2017 (about $1668-4078 \mathrm{~m}$ ) and Akpabio et al 2014 about 2050 - $11620 \mathrm{ft}(624.84-3541.78 \mathrm{~m})$. The hydrocarbon reservoirs were found to be in the Agbada formation (approximately $1000-4000 \mathrm{~m}$, Inyang et al 2015) which is in conformity with the geology of the Niger Delta, Nigeria, they have a Net Pay thickness

\begin{tabular}{|c|c|c|c|c|c|c|c|c|c|c|c|c|c|}
\hline \multirow[b]{2}{*}{ Curve } & \multirow[b]{2}{*}{$\begin{array}{l}\text { Uni } \\
\text { ts }\end{array}$} & \multicolumn{3}{|c|}{$\begin{array}{l}\text { Well A, Top:5514.5ft,Bot- } \\
\text { tom:6980,Net:1466ft }\end{array}$} & \multicolumn{3}{|c|}{$\begin{array}{l}\text { Well A,R1, Top:5514.5ft,Bot- } \\
\text { tom:5643.5ft,Net: } 129.5 \mathrm{ft}\end{array}$} & \multicolumn{3}{|c|}{$\begin{array}{l}\text { Well A, R2 Top:584ft,Bot- } \\
\text { tom:6017ft,Net: } 169.5 \mathrm{ft}\end{array}$} & \multicolumn{3}{|c|}{$\begin{array}{l}\text { Well A, R3 Top:6160ft,Bot- } \\
\text { tom6333.5ft,Net: } 176 \mathrm{ft}\end{array}$} \\
\hline & & Min & Max & Mean & Min & Max & Mean & Min & Max & Mean & Min & Max & Mean \\
\hline AI & Psi & $\begin{array}{l}10786 . \\
85\end{array}$ & $\begin{array}{l}44679 . \\
33\end{array}$ & $\begin{array}{l}18941 . \\
07\end{array}$ & $\begin{array}{l}16039.6 \\
1\end{array}$ & 26468.77 & $\begin{array}{l}17992.6 \\
8\end{array}$ & $\begin{array}{l}15437 . \\
91\end{array}$ & $\begin{array}{l}20269.3 \\
3\end{array}$ & $\begin{array}{l}17877 . \\
25\end{array}$ & $\begin{array}{l}13244 . \\
39\end{array}$ & $\begin{array}{l}23290.4 \\
7\end{array}$ & $\begin{array}{l}19180 . \\
44\end{array}$ \\
\hline BVW & $\begin{array}{l}\mathrm{De} \\
\mathrm{c}\end{array}$ & 0.00 & 0.30 & 0.12 & 0.08 & 0.18 & 0.10 & 0.10 & 0.16 & 0.13 & 0.02 & 0.18 & 0.11 \\
\hline CAL & $\begin{array}{l}\text { inc } \\
h\end{array}$ & 10.54 & 20.15 & 12.96 & 11.30 & 15.94 & 13.35 & 10.59 & 13.87 & 11.89 & 11.47 & 17.42 & 13.80 \\
\hline $\begin{array}{l}\text { DTsE } \\
\text { mp }\end{array}$ & $\begin{array}{l}\mathrm{uSe} \\
\mathrm{c} / \mathrm{ft}\end{array}$ & 76.61 & 347.93 & 236.84 & 129.41 & 304.70 & 255.92 & 216.80 & 295.65 & 249.51 & 169.85 & 294.78 & 226.59 \\
\hline $\mathrm{E}$ & Psi & 3.28 & 74.52 & 10.02 & 5.87 & 27.65 & 8.44 & 5.96 & 11.58 & 8.59 & 5.34 & 17.87 & 10.46 \\
\hline EI_10 & & $\begin{array}{l}2673.6 \\
3\end{array}$ & $\begin{array}{l}10275 . \\
69\end{array}$ & $\begin{array}{l}4559.9 \\
5\end{array}$ & 3910.53 & 6226.87 & 4348.63 & $\begin{array}{l}3762.8 \\
9\end{array}$ & 4860.74 & $\begin{array}{l}4319.0 \\
3\end{array}$ & $\begin{array}{l}3241.7 \\
1\end{array}$ & 5530.15 & $\begin{array}{l}4611.8 \\
1\end{array}$ \\
\hline EI_20 & & $\begin{array}{l}1474.0 \\
4\end{array}$ & $\begin{array}{l}4567.0 \\
1\end{array}$ & $\begin{array}{l}2312.2 \\
2\end{array}$ & 2055.95 & 2953.93 & 2229.81 & $\begin{array}{l}1974.5 \\
9\end{array}$ & 2436.73 & $\begin{array}{l}2212.0 \\
3\end{array}$ & $\begin{array}{l}1723.6 \\
5\end{array}$ & 2694.31 & $\begin{array}{l}2329.7 \\
9\end{array}$ \\
\hline EI_30 & & 592.01 & $\begin{array}{l}1318.4 \\
5\end{array}$ & 817.36 & 761.54 & 942.33 & 801.55 & 720.92 & 845.95 & 793.64 & 654.87 & 895.35 & 818.58 \\
\hline $\begin{array}{l}\text { GR } \\
\text { NM }\end{array}$ & $\begin{array}{l}\text { gA } \\
\text { PI }\end{array}$ & 27.22 & 107.51 & 48.39 & 27.78 & 71.17 & 36.26 & 30.66 & 58.44 & 38.49 & 27.31 & 84.65 & 35.46 \\
\hline $\mathrm{KB}$ & $\begin{array}{l}\mathrm{KB} \\
\text { ars }\end{array}$ & 5.52 & 32.80 & 10.66 & 8.46 & 17.27 & 9.91 & 8.24 & 11.59 & 9.89 & 7.10 & 14.15 & 10.87 \\
\hline LL9D & $\begin{array}{l}\text { oh } \\
\text { ms }\end{array}$ & 1.906 & 69.823 & 12.714 & 7.311 & 33.729 & 21.605 & 9.290 & 19.231 & 14.318 & 8.790 & 27.040 & 17.549 \\
\hline $\mathrm{Mu}$ & $\begin{array}{l}\mathrm{KB} \\
\text { ars }\end{array}$ & 1.172 & 33.558 & 3.742 & 2.119 & 11.208 & 3.113 & 2.160 & 4.342 & 3.171 & 1.943 & 6.936 & 3.907 \\
\hline NPHI & dec & 0.000 & 0.000 & 0.000 & 0.000 & 0.000 & 0.000 & 0.000 & 0.000 & 0.000 & 0.000 & 0.000 & 0.000 \\
\hline PHI & $\begin{array}{l}\text { De } \\
\text { c }\end{array}$ & 0.000 & 0.600 & 0.472 & 0.190 & 0.600 & 0.589 & 0.450 & 0.600 & 0.598 & 0.019 & 0.600 & 0.588 \\
\hline $\begin{array}{l}\text { Pois- } \\
\text { Ratio }\end{array}$ & & 0.110 & 0.401 & 0.346 & 0.233 & 0.384 & 0.359 & 0.333 & 0.380 & 0.356 & 0.289 & 0.380 & 0.340 \\
\hline
\end{tabular}
between $63.5 \mathrm{ft}(19.05 \mathrm{~m})$ to $176 \mathrm{ft}(52.8 \mathrm{~m})$ showing that most of the reservoirs are of economic importance.

Table 2: Elastic and Petrophysical Analysis for Well A and Three Reservoirs in Well A 


\begin{tabular}{|c|c|c|c|c|c|c|c|c|c|c|c|c|c|}
\hline $\begin{array}{l}\text { RHO } \\
\text { B }\end{array}$ & $\begin{array}{l}\mathrm{gm} / \\
\mathrm{cc}\end{array}$ & 1.527 & 2.494 & 2.147 & 2.020 & 2.254 & 2.146 & 1.848 & 2.220 & 2.105 & 1.681 & 2.265 & 2.129 \\
\hline SI & Psi & $\begin{array}{l}4387.6 \\
51\end{array}$ & $\begin{array}{l}28926 . \\
164\end{array}$ & $\begin{array}{l}9203.4 \\
21\end{array}$ & $\begin{array}{l}6951.05 \\
9\end{array}$ & $\begin{array}{l}15612.10 \\
8\end{array}$ & $\begin{array}{l}8442.10 \\
5\end{array}$ & $\begin{array}{l}6842.9 \\
01\end{array}$ & $\begin{array}{l}10131.7 \\
24\end{array}$ & $\begin{array}{l}8464.9 \\
69\end{array}$ & $\begin{array}{l}5929.5 \\
11\end{array}$ & $\begin{array}{l}12681.0 \\
99\end{array}$ & $\begin{array}{l}9445.5 \\
52\end{array}$ \\
\hline $\begin{array}{l}\text { SONI } \\
\mathrm{C}\end{array}$ & $\begin{array}{l}\text { us/f } \\
\mathrm{t}\end{array}$ & 50.700 & $\begin{array}{l}141.52 \\
4\end{array}$ & $\begin{array}{l}114.02 \\
3\end{array}$ & 76.328 & 132.048 & 119.587 & $\begin{array}{l}108.37 \\
1\end{array}$ & 129.904 & $\begin{array}{l}117.90 \\
0\end{array}$ & 92.480 & 129.694 & $\begin{array}{l}111.25 \\
6\end{array}$ \\
\hline SW & $\begin{array}{l}\text { De } \\
\mathrm{c}\end{array}$ & 0.127 & 1.000 & 0.384 & 0.127 & 0.715 & 0.175 & 0.174 & 0.324 & 0.209 & 0.144 & 1.000 & 0.197 \\
\hline VCL & $\begin{array}{l}\text { De } \\
\text { c }\end{array}$ & 0.000 & 0.637 & 0.134 & 0.000 & 0.326 & 0.033 & 0.000 & 0.217 & 0.048 & 0.000 & 0.441 & 0.026 \\
\hline $\mathrm{Vp}$ & $\begin{array}{l}\mathrm{ft} / \mathrm{s} \\
\mathrm{ec}\end{array}$ & $\begin{array}{l}7065.9 \\
29\end{array}$ & $\begin{array}{l}19723 . \\
865\end{array}$ & $\begin{array}{l}8822.1 \\
65\end{array}$ & $\begin{array}{l}7572.99 \\
8\end{array}$ & $\begin{array}{l}13101.40 \\
4\end{array}$ & $\begin{array}{l}8383.13 \\
4\end{array}$ & $\begin{array}{l}7698.0 \\
22\end{array}$ & $\begin{array}{l}9227.59 \\
5\end{array}$ & $\begin{array}{l}8490.2 \\
92\end{array}$ & $\begin{array}{l}7710.4 \\
39\end{array}$ & $\begin{array}{l}10813.1 \\
61\end{array}$ & $\begin{array}{l}9000.9 \\
99\end{array}$ \\
\hline $\begin{array}{l}\text { VpVs } \\
\text { Ratio }\end{array}$ & & 1.511 & 2.458 & 2.071 & 1.695 & 2.308 & 2.137 & 2.001 & 2.276 & 2.115 & 1.837 & 2.273 & 2.035 \\
\hline Vs & $\begin{array}{l}\mathrm{ft} / \mathrm{s} \\
\mathrm{ec}\end{array}$ & $\begin{array}{l}2874.1 \\
33 \\
\end{array}$ & $\begin{array}{l}13053 . \\
138\end{array}$ & $\begin{array}{l}4286.4 \\
27\end{array}$ & $\begin{array}{l}3281.89 \\
7\end{array}$ & 7727.619 & $\begin{array}{l}3933.37 \\
5\end{array}$ & $\begin{array}{l}3382.4 \\
36\end{array}$ & $\begin{array}{l}4612.45 \\
8\end{array}$ & $\begin{array}{l}4019.5 \\
48\end{array}$ & $\begin{array}{l}3392.4 \\
21\end{array}$ & $\begin{array}{l}5887.50 \\
6\end{array}$ & $\begin{array}{l}4430.2 \\
38\end{array}$ \\
\hline
\end{tabular}

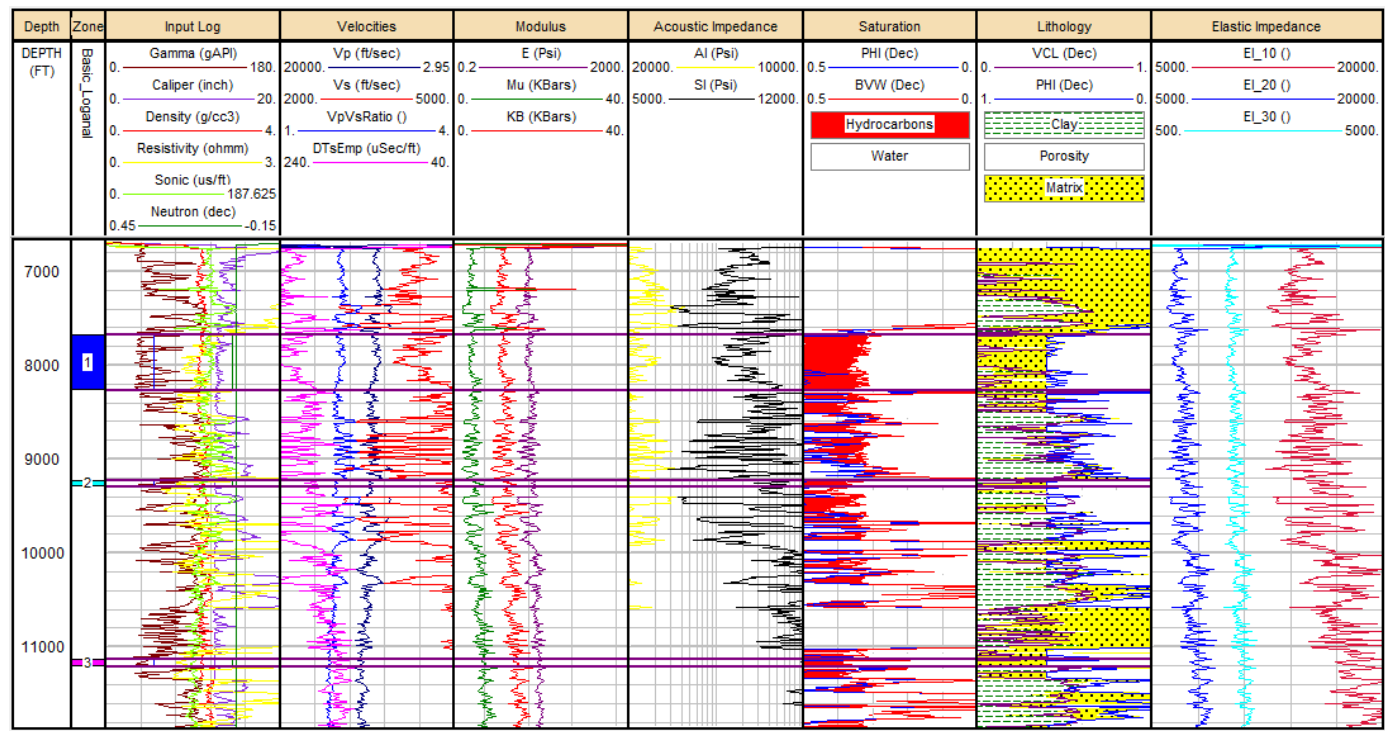

Fig. 1: Log Plots of Elastic and Petrophysical Properties for Well A.

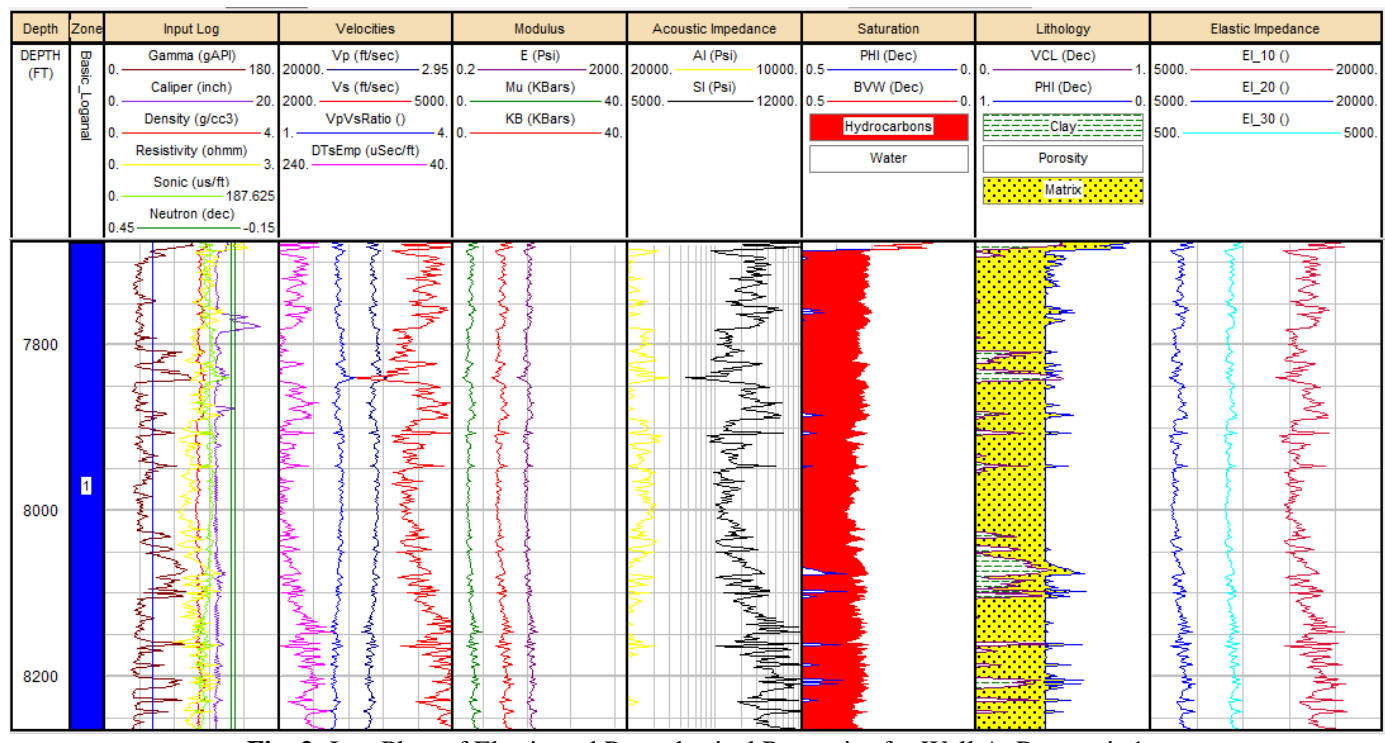

Fig. 2: Log Plots of Elastic and Petrophysical Properties for Well A, Reservoir 1. 


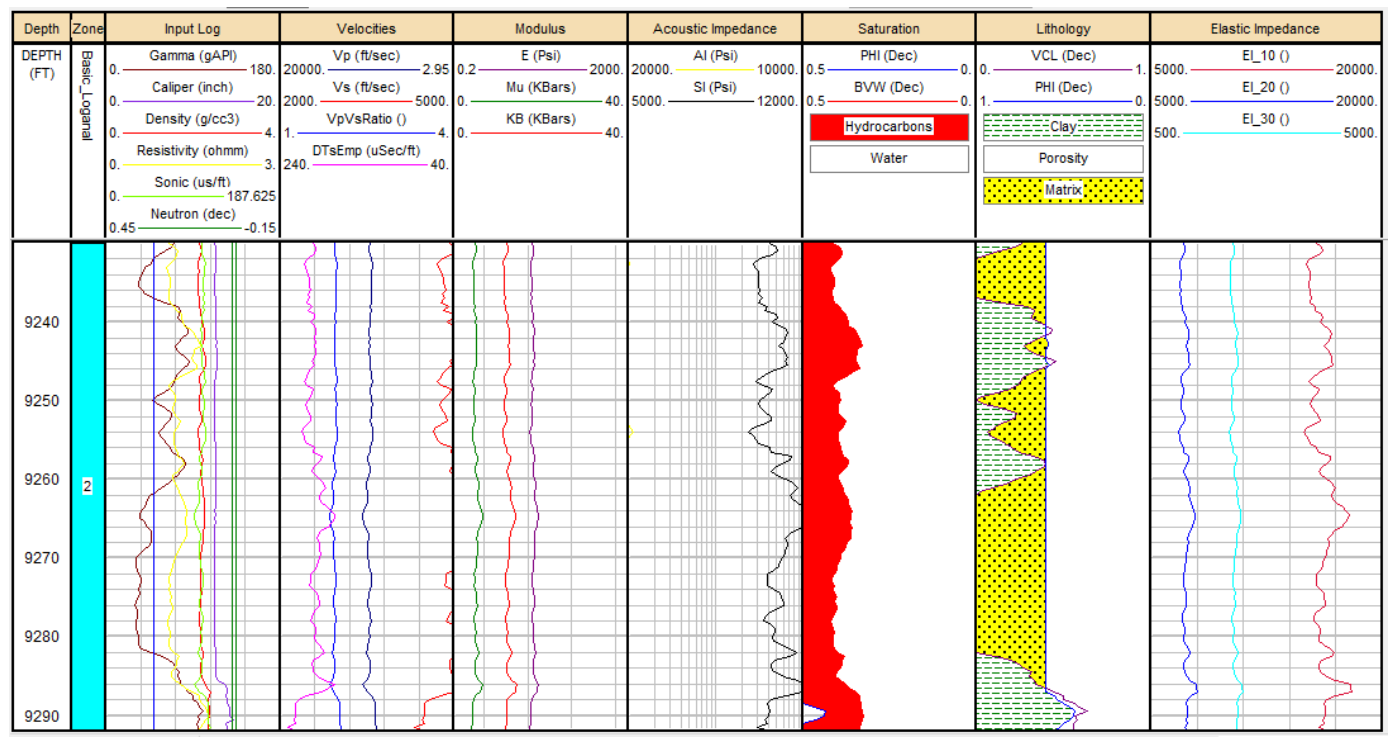

Fig. 3: Log Plots of Elastic and Petrophysical Properties for Well A, Reservoir 2.

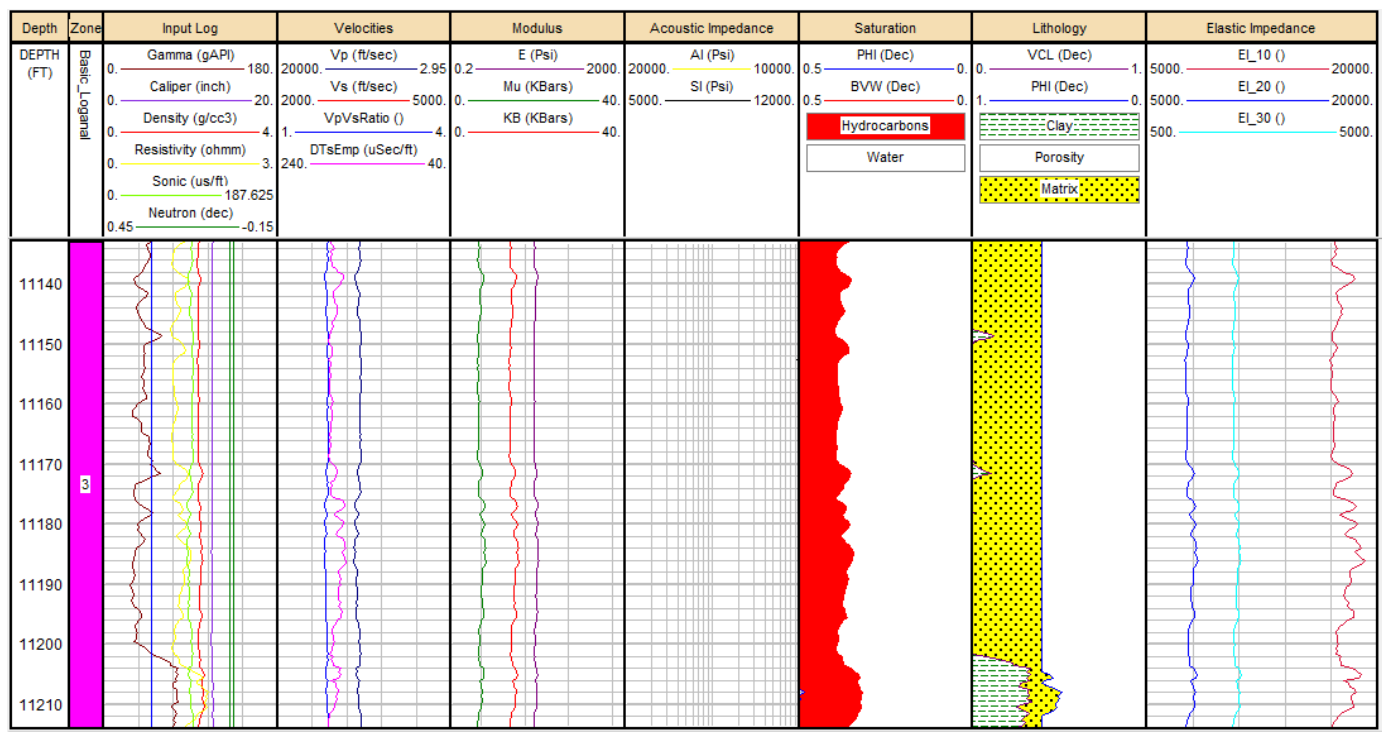

Fig. 4: Log Plots of Elastic and Petrophysical Properties for Well A, Reservoir 3.

Table 3: Elastic and Petrophysical Analysis for Well B and Three Reservoirs in Well B

\begin{tabular}{|c|c|c|c|c|c|c|c|c|c|c|c|c|c|}
\hline \multirow[b]{2}{*}{$\begin{array}{l}\text { Curv } \\
\mathrm{e}\end{array}$} & \multirow[b]{2}{*}{$\begin{array}{l}\text { Un } \\
\text { its }\end{array}$} & \multicolumn{3}{|c|}{$\begin{array}{l}\text { Well B, Top: } 7803 \mathrm{ft} \text {, Bottom: } \\
11507 \mathrm{ft} \text {, Net: } 3694.5 \mathrm{ft}\end{array}$} & \multicolumn{3}{|c|}{$\begin{array}{l}\text { Well B, R1, Top: } 9663 \mathrm{ft} \text {, Bot- } \\
\text { tom: } 9800 \mathrm{ft} \text {, Net: } 137.7 \mathrm{ft}\end{array}$} & \multicolumn{3}{|c|}{$\begin{array}{l}\text { Well B, R2, Top: } 10000 \mathrm{ft} \text {, Bot- } \\
\text { tom: } 10125 \mathrm{ft} \text {, Net: } 125.5 \mathrm{ft}\end{array}$} & \multicolumn{3}{|c|}{$\begin{array}{l}\text { Well B, R3, Top: } 11444 \mathrm{ft} \text {, Bot- } \\
\text { tom: } 11507 \mathrm{ft} \text {, Net: } 63.5 \mathrm{ft}\end{array}$} \\
\hline & & Min & Max & Mean & Min & Max & Mean & Min & Max & Mean & Min & Max & Mean \\
\hline AI & Psi & $\begin{array}{l}17985 \\
.611\end{array}$ & $\begin{array}{l}45256.5 \\
47\end{array}$ & $\begin{array}{l}24846 . \\
127\end{array}$ & $\begin{array}{l}20462.3 \\
36\end{array}$ & $\begin{array}{l}36742.1 \\
48\end{array}$ & $\begin{array}{l}24131.6 \\
97\end{array}$ & $\begin{array}{l}24362.4 \\
51\end{array}$ & $\begin{array}{l}31522.2 \\
75\end{array}$ & $\begin{array}{l}26602.3 \\
79\end{array}$ & $\begin{array}{l}26843.3 \\
50\end{array}$ & $\begin{array}{l}32908.1 \\
25\end{array}$ & $\begin{array}{l}29562.3 \\
20\end{array}$ \\
\hline $\begin{array}{l}\text { BV } \\
\text { W }\end{array}$ & $\begin{array}{l}\text { De } \\
\mathrm{c}\end{array}$ & 0.000 & 0.566 & 0.092 & 0.000 & 0.232 & 0.032 & N/A & N/A & N/A & N/A & N/A & N/A \\
\hline CAL & $\begin{array}{l}\text { inc } \\
\mathrm{h}\end{array}$ & & 12.994 & 7.288 & 10.482 & 11.629 & 10.872 & 8.502 & 9.988 & 8.600 & 8.245 & 8.442 & 8.349 \\
\hline $\begin{array}{l}\text { DTs } \\
\text { Emp }\end{array}$ & $\begin{array}{l}\mathrm{uS} \\
\mathrm{ec} / \\
\mathrm{ft}\end{array}$ & $\begin{array}{l}83.09 \\
5\end{array}$ & 239.592 & $\begin{array}{l}174.83 \\
4\end{array}$ & 114.896 & 208.409 & 182.708 & 138.683 & 168.958 & 155.491 & 122.009 & 151.826 & 136.071 \\
\hline E & Psi & 9.089 & 74.575 & 19.146 & 12.657 & 43.596 & 17.326 & 18.825 & 30.374 & 22.708 & 23.592 & 35.672 & 29.371 \\
\hline EI_1 & & 4338. & 10399.2 & 5888.0 & 4898.01 & 8538.10 & 5731.22 & 5775.88 & 7385.67 & 6278.32 & 6329.84 & 7686.29 & 6936.24 \\
\hline 0 & & 249 & 57 & 94 & 7 & 0 & 6 & 1 & 1 & 4 & 6 & 5 & 2 \\
\hline EI_2 & & 2211. & 4610.19 & 2853.3 & 2442.46 & 3909.46 & 2794.44 & 2797.05 & 3462.91 & 3005.95 & 3019.35 & 3571.55 & 3265.54 \\
\hline $0^{-}$ & & 541 & 0 & 50 & 9 & 2 & 4 & 3 & 4 & 4 & 4 & 3 & 4 \\
\hline $\begin{array}{l}\text { EI_3 } \\
0\end{array}$ & & $\begin{array}{l}780.9 \\
40\end{array}$ & $\begin{array}{l}1325.73 \\
6\end{array}$ & $\begin{array}{l}941.01 \\
9\end{array}$ & 841.095 & $\begin{array}{l}1181.31 \\
2\end{array}$ & 929.998 & 919.651 & $\begin{array}{l}1085.08 \\
5\end{array}$ & 972.676 & 970.894 & $\begin{array}{l}1103.78 \\
9\end{array}$ & $\begin{array}{l}1029.77 \\
9\end{array}$ \\
\hline $\begin{array}{l}\mathrm{GR}_{-} \\
\mathrm{NM}\end{array}$ & $\begin{array}{l}\mathrm{gA} \\
\text { PI }\end{array}$ & & 134.423 & 74.397 & 29.448 & 107.970 & 76.050 & 30.001 & 129.819 & 76.381 & 30.813 & 70.338 & 47.641 \\
\hline KB & $\begin{array}{l}\mathrm{KB} \\
\text { ars }\end{array}$ & $\begin{array}{l}10.07 \\
6\end{array}$ & $\begin{array}{l}3324175 \\
04.000\end{array}$ & $\begin{array}{l}89734 \\
9.370\end{array}$ & 11.883 & 24.779 & 14.440 & 14.820 & 20.140 & 16.544 & 16.827 & 21.596 & 19.053 \\
\hline $\begin{array}{l}\text { LL9 } \\
\text { D }\end{array}$ & $\begin{array}{l}\text { oh } \\
\text { ms }\end{array}$ & & 275.423 & & 1.180 & 172.187 & 10.676 & & & & & & \\
\hline $\mathrm{Mu}$ & $\begin{array}{l}\mathrm{KB} \\
\text { ars }\end{array}$ & & 32.991 & & 4.785 & 18.063 & 6.677 & 7.306 & 12.168 & 8.933 & 9.310 & 14.616 & 11.819 \\
\hline
\end{tabular}




\begin{tabular}{|c|c|c|c|c|c|c|c|c|c|c|c|c|c|}
\hline $\begin{array}{l}\text { NPH } \\
\text { I }\end{array}$ & dec & & 52.006 & & 16.120 & 50.705 & 32.933 & & & & & & \\
\hline PHI & $\begin{array}{l}\text { De } \\
\mathrm{c}\end{array}$ & 0.000 & 0.600 & 0.112 & 0.000 & 0.574 & 0.100 & N/A & N/A & N/A & N/A & N/A & N/A \\
\hline $\begin{array}{l}\text { Pois- } \\
\text { Ratio }\end{array}$ & & 0.130 & 0.350 & 0.292 & 0.207 & 0.327 & 0.302 & 0.248 & 0.288 & 0.272 & 0.220 & 0.267 & 0.244 \\
\hline $\begin{array}{l}\text { RHO } \\
\text { B }\end{array}$ & $\begin{array}{l}\mathrm{gm} \\
/ \mathrm{cc}\end{array}$ & & 2.589 & & 2.123 & 2.584 & 2.330 & 2.184 & 2.547 & 2.312 & 2.242 & 2.474 & 2.335 \\
\hline SI & Psi & $\begin{array}{l}8683 . \\
095\end{array}$ & $\begin{array}{l}29508.2 \\
77\end{array}$ & $\begin{array}{l}13486 . \\
832\end{array}$ & $\begin{array}{l}10474.2 \\
22\end{array}$ & $\begin{array}{l}22339.2 \\
56\end{array}$ & $\begin{array}{l}12862.9 \\
11\end{array}$ & $\begin{array}{l}13287.3 \\
37\end{array}$ & $\begin{array}{l}18196.9 \\
63\end{array}$ & $\begin{array}{l}14900.5 \\
73\end{array}$ & $\begin{array}{l}15172.8 \\
59\end{array}$ & $\begin{array}{l}19516.3 \\
95\end{array}$ & $\begin{array}{l}17214.8 \\
16\end{array}$ \\
\hline $\begin{array}{l}\text { SON } \\
\text { IC }\end{array}$ & $\begin{array}{l}\mathrm{us} / \\
\mathrm{ft}\end{array}$ & & 115.180 & 90.979 & 69.857 & 105.723 & 96.896 & 80.266 & 92.150 & 87.015 & 73.078 & 85.599 & 79.131 \\
\hline SW & $\begin{array}{l}\text { De } \\
\mathrm{c}\end{array}$ & 0.040 & 1.000 & 0.959 & 0.060 & 1.000 & 0.836 & N/A & N/A & N/A & N/A & N/A & N/A \\
\hline VCL & $\begin{array}{l}\mathrm{De} \\
\mathrm{c}\end{array}$ & 0.000 & 0.657 & 0.369 & 0.000 & 0.641 & 0.369 & N/A & N/A & N/A & N/A & N/A & N/A \\
\hline$V p$ & $\begin{array}{l}\mathrm{ft} / \mathrm{s} \\
\mathrm{ec}\end{array}$ & $\begin{array}{l}8682 . \\
063\end{array}$ & $\begin{array}{l}18456.9 \\
94\end{array}$ & $\begin{array}{l}10737 . \\
591\end{array}$ & $\begin{array}{l}9458.65 \\
3\end{array}$ & $\begin{array}{l}14314.9 \\
37\end{array}$ & $\begin{array}{l}10369.2 \\
95\end{array}$ & $\begin{array}{l}10851.8 \\
72\end{array}$ & $\begin{array}{l}12458.5 \\
75\end{array}$ & $\begin{array}{l}11499.2 \\
41\end{array}$ & $\begin{array}{l}11682.3 \\
80\end{array}$ & $\begin{array}{l}13684.0 \\
09\end{array}$ & $\begin{array}{l}12654.5 \\
44\end{array}$ \\
\hline $\begin{array}{l}\text { VpV } \\
\text { sRati } \\
\text { o }\end{array}$ & & 1.534 & 2.080 & 1.854 & 1.645 & 1.971 & 1.882 & 1.728 & 1.834 & 1.786 & 1.670 & 1.774 & 1.719 \\
\hline Vs & $\begin{array}{l}\mathrm{ft} / \mathrm{s} \\
\mathrm{ec}\end{array}$ & $\begin{array}{l}4173 . \\
762\end{array}$ & $\begin{array}{l}12034.3 \\
71\end{array}$ & $\begin{array}{l}5826.7 \\
36\end{array}$ & $\begin{array}{l}4798.26 \\
6\end{array}$ & $\begin{array}{l}8703.49 \\
3\end{array}$ & $\begin{array}{l}5530.56 \\
7\end{array}$ & $\begin{array}{l}5918.63 \\
6\end{array}$ & $\begin{array}{l}7210.68 \\
3\end{array}$ & $\begin{array}{l}6439.22 \\
4\end{array}$ & $\begin{array}{l}6586.49 \\
7\end{array}$ & $\begin{array}{l}8196.12 \\
6\end{array}$ & $\begin{array}{l}7368.27 \\
2\end{array}$ \\
\hline
\end{tabular}

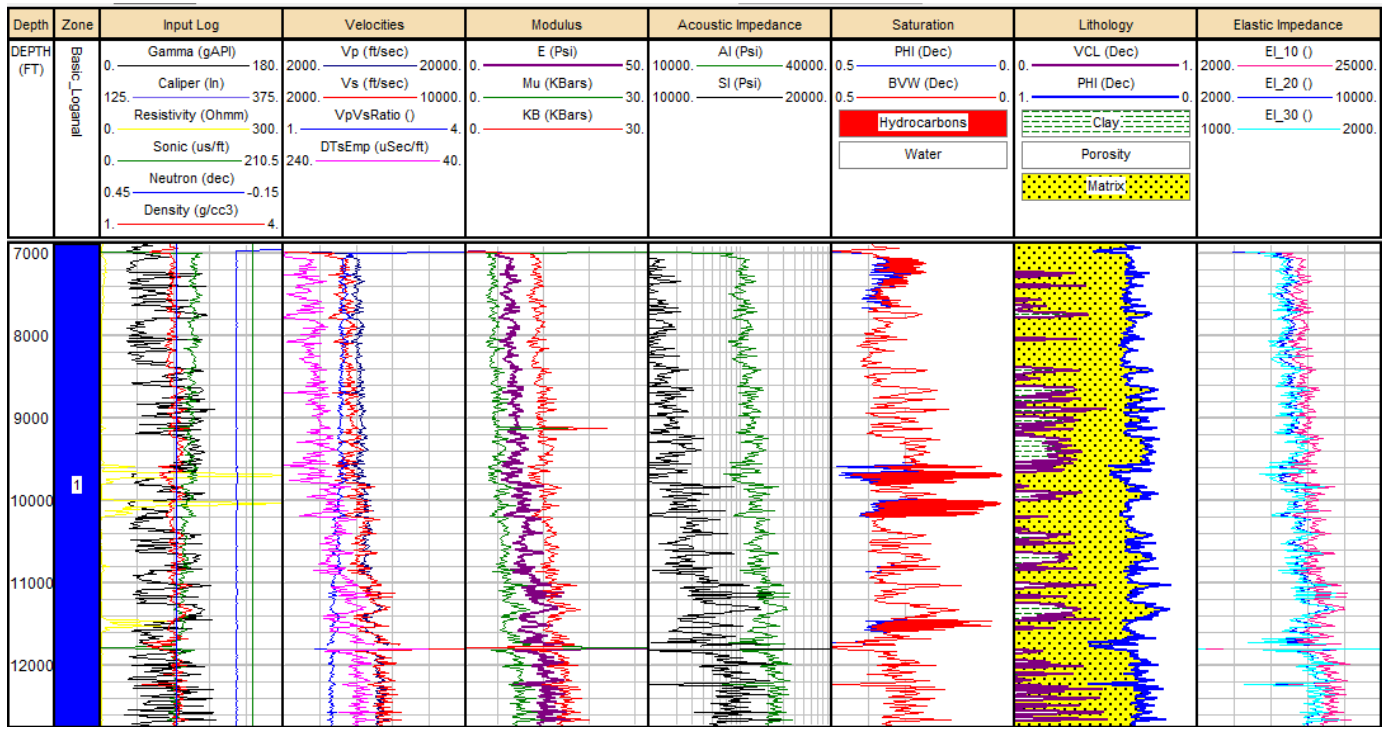

Fig. 5: Log Plots of Elastic and Petrophysical Properties for Well B.

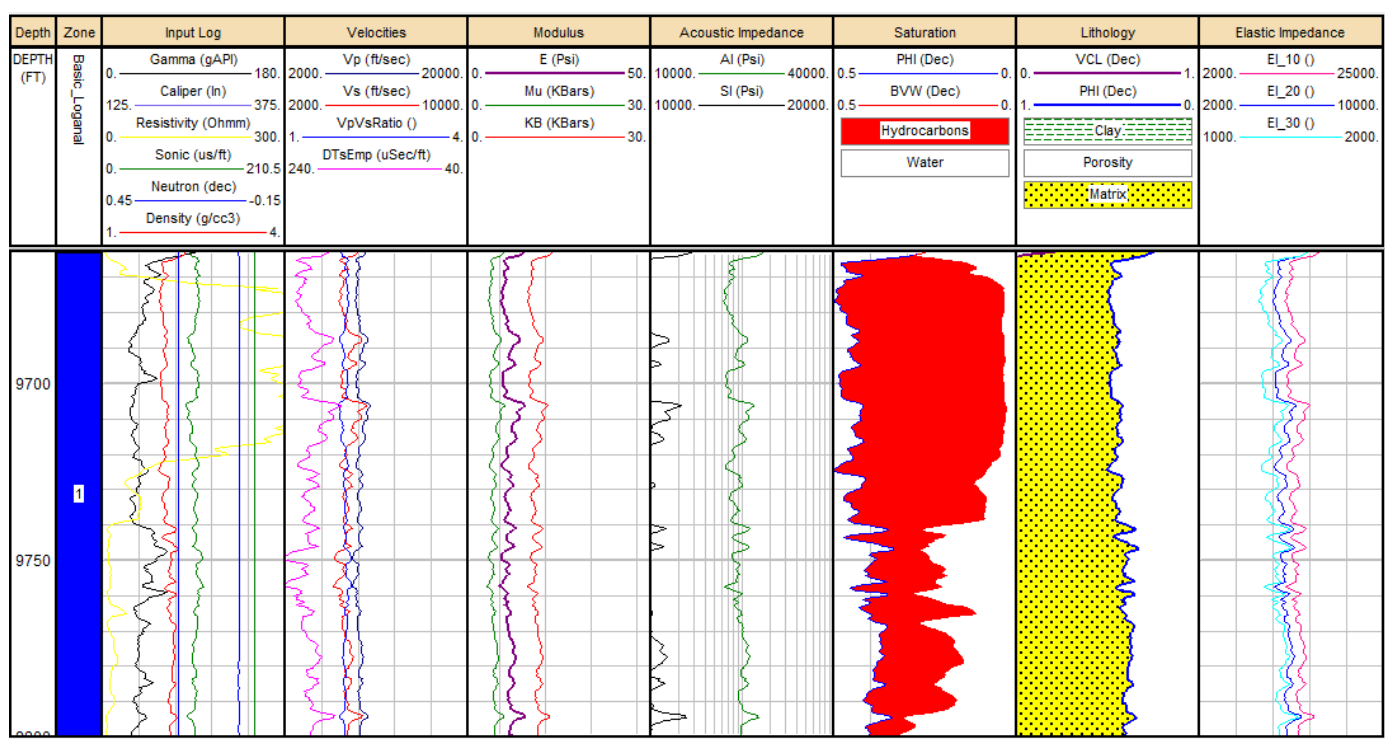

Fig. 6: Log Plots of Elastic and Petrophysical Properties for Well B, Reservoir 1. 


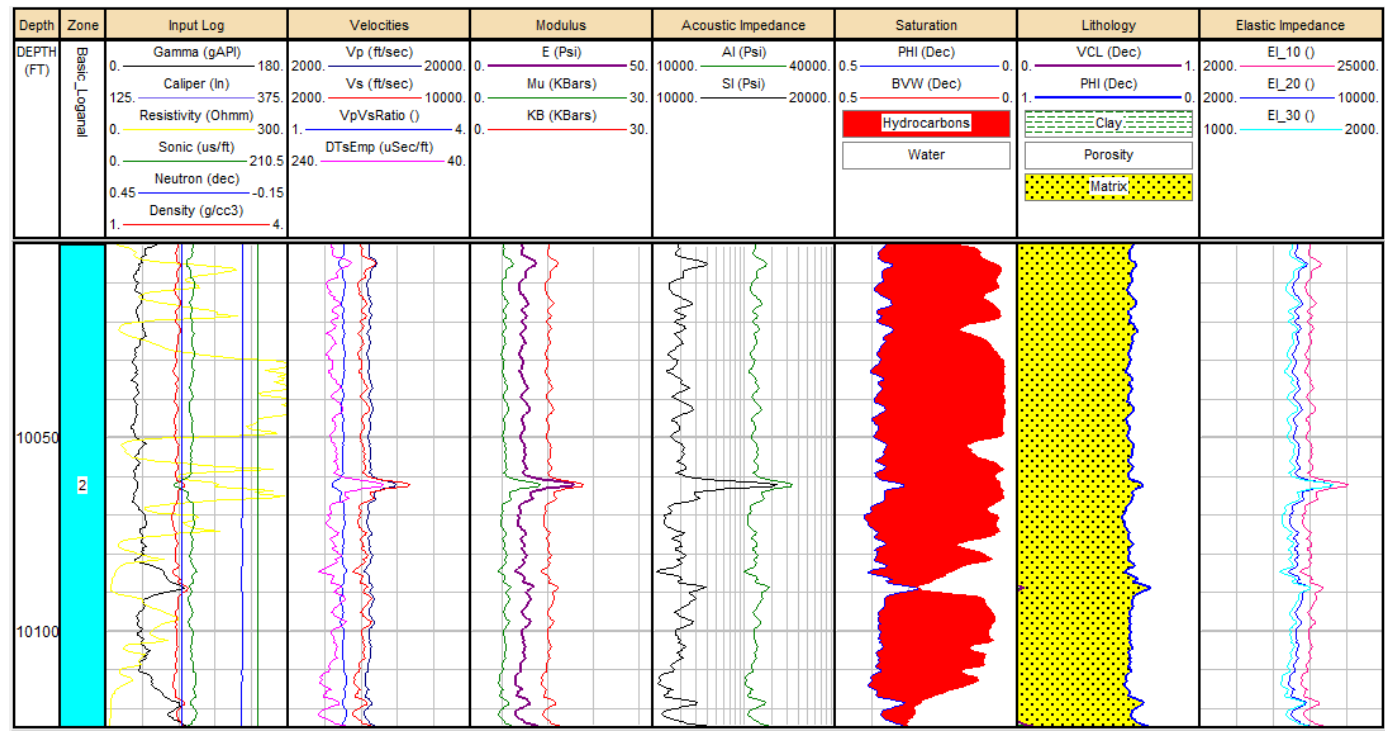

Fig. 7: Log Plots of Elastic and Petrophysical Properties for Well B, Reservoir 2.

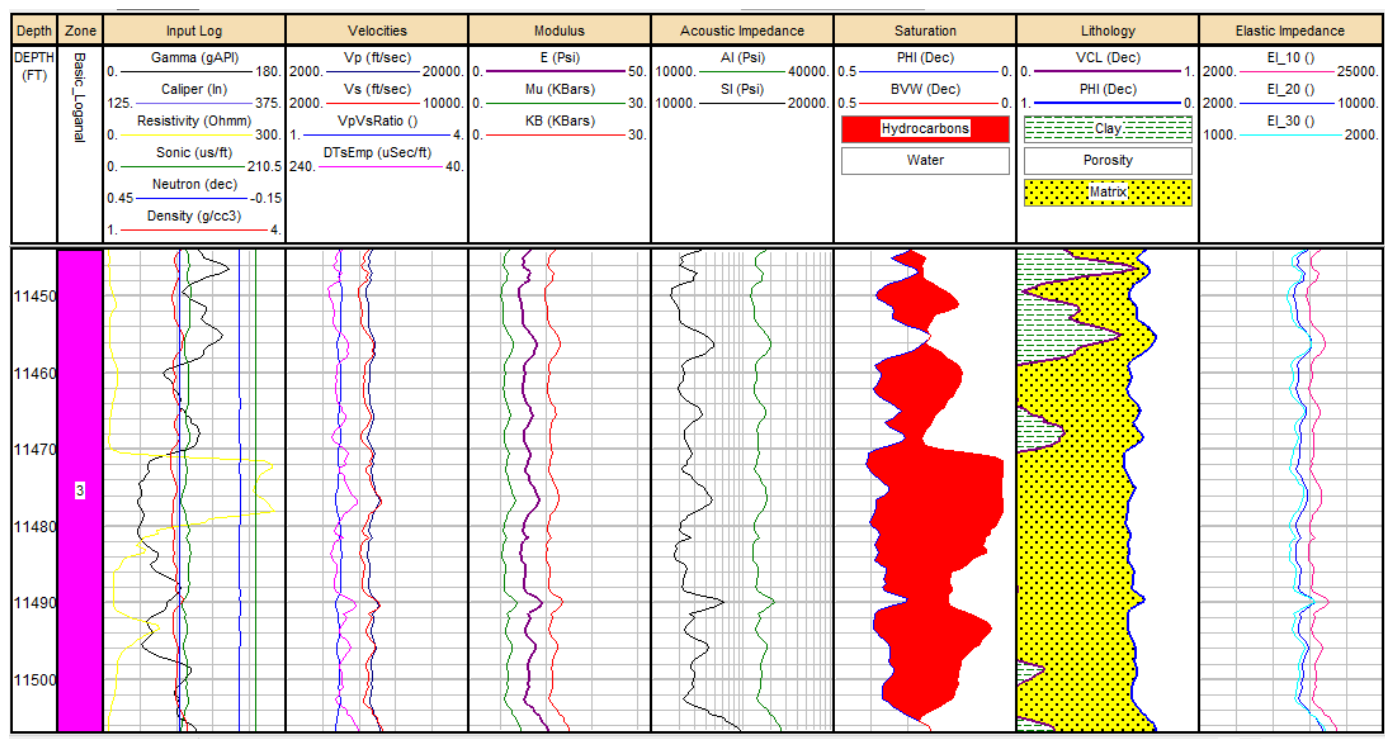

Fig. 8: Log Plots of Elastic and Petrophysical Properties for Well B, Reservoir 3.

The reservoirs in the study area falls within the Agbada Formation whose stratigraphic succession consists of interbedded sandstones and shales. Typical elastic and rock strength properties derived from empirical relations are summarized in Tables 1 and 2 . There are significant variations in properties between the cap rocks and the reservoir sand units across the field. The cap rock which is shale, has high Poison ratio, elastic, bulk and rigidity moduli. However, lower bulk compressibility and rock strength makes the shale more ductile, stiffer, less compressible and more prone to compressive shear failure, but better fracture stimulation barriers.

Conversely, sandstones, the main reservoir rocks, have relatively lower Poisson ratio, elastic, bulk and rigidity moduli but higher compressibility and rock strength making them more brittle with higher potential for tensile failure. Thus, sandstone will fracture before shales in a hydraulic fracture stimulation process under the same fracture gradient while shales will form the barrier to fracture growth. Low rock strength accounts for the occurrence of wellbore failures in shales and weak shaly sandstones. The Elastic properties of the reservoirs that are found mostly in the sandstone lithology varies between 15437.91 to $31522.28 \mathrm{psi}, 7.10$ to $24.78 \mathrm{Kbar}$, 5929.51 to $22339.26 \mathrm{psi}, 1.94$ to $18.06 \mathrm{Kbar}, 1.70$ to $2.31,5.34$ to 43.59psi of Acoustic Impedance, Bulk Modulus, Shear Impedance, Shear Modulus, Velocity ratio and Young Modulus respectively. There is a general decreasing trend in the modulus of rigidity, bulk and matrix moduli and an increase in elastic modulus of the rocks with depth. Compaction equilibrium during diagenesis under anoxic conditions depicted by normally pressured shale source beds favoured hydrocarbon accumulation with the shale smears on the faults and caps on the sand tops providing the traps. The increase in rock compressibility with effective vertical stress and effective porosity and decrease in compressibility with depth and decrease in effective porosity with bulk compressibility further support equilibrium compaction. Increase in effective overburden stress due to sediment loading and fluids expulsion causes grain sliding in shear and compactional deformation with reduction in the bulk and grain compressibility and pore volume of the sediment with increasing depth. Grain to grain contact destroys the cement bonds and closes packing of individual grains by elastic distortions and strains. This mechanism is responsible for generation of over pressures since impermeable sediments such as shales saturated with an incompressible fluid will not deform elastically and when there is disequilibrium compaction, abnormal pore pressures will form as reported in most fields in the Niger Delta. Young tertiary sedimentary rocks deform primarily by compaction resulting in progressive loss of porosity with increasing depth of burial.

\section{Conclusion}

Well log data have been used to determine elastic parameters of five wells in the Niger Delta Region of Nigeria. Elastic properties of rocks are affected by some geological factors which include: Depth of burial, Lithology, Anisotropy and Diastrophism. The specific transit times are influenced by these geological factors 
as well as porosity. Texture and geological history determine the elastic properties more than the mineral composition. Crystalline rocks generally exhibit larger values of elastic moduli than fragmental rocks. From the analysis of Elastic parameters of the wells geomechanical modelling, wellbore stability analysis and sanding, which can be applied in practical situation to optimize drilling, completion and production of wells can easily be achieved.

\section{References}

[1] Anderson P.F., ( 2009). Comparing post-stack AVO inversion to pre-stack inversion for estimating rock properties, Frontiers Innovation, CSPG, CSEG, CWLS Convention, Canada.

[2] Agbasi, E. O., Inyang, N. and Ibout, J. (2013), Estimation of Water Saturation in Niger Delta Nigeria Using Wire-Line logs. IOSR, Journal of Applied Physics (IOSR-JAP). E-ISSN: 2278 - 4861, volume 3 , issue 4(2013) pp.66 -71. www.iosrjournals.org

[3] Akpabio, I., Ibuot, J., Agbasi, O. E and Ojo, O. T. (2014), Petrophys ical Characterization of eight wells from Wire-line Logs, Niger Delta Nigeria. Asian Journal of Applied Science, ISSN 2521 - 0893, volume 02, Issue 02, $105-109$.

[4] Akankpo, A.O., Umoren E.B., and Agbasi O.E., (2015). Porosity Estimation Using Wire-Line Log to Depth in Niger Delta, Nigeria. IOSR Journal of Applied Geology and Geophysics (IOSR-JAGG) eISSN: 2321-0990, p-ISSN: 2321-0982.Volume 3, Issue 4 Ver. II (Jul - Aug. 2015), PP 31-38 www.iosrjournals.org.

[5] Agbasi. O. E, Akankpo A. O., and Essien, U. E. (2017). Estimation of Reservoir Potentials of Two Wells in Niger Delta Region, Nigeria Journal of Geosciences and Geomatics, 2017, Vol. 5, No. 2, 87-95. DOI: $10.12691 /$ jgg-5-2-5.

[6] Agbasi, E. Okechukwu, Godwill U. Chukwu, Magnus U. Igboekwe and Edet E. Sunday. (2018). Pore Fluid and Lithology Discrimination of a Well in the Niger Delta Region using Elastic Parameters. World News of Natural Science. WNOFNS 17 (2018) 75-88.

[7] Bassey, C.E., Harry,T.A and Ibidapo, A.S (2013): Sequence stratigraphic study of UNIBR field in the northern delta depobelt of Nige Delta, Nigeria. Journal of Environment and earth science. pg. 44-45

[8] Connolly P., (1999). Elastic Impedance: The Leading Edge, v. 18, p. 438-452. https://doi.org/10.1190/1.1438307.

[9] Cooke D. A. and Schneider W.A., (1983). Generalized inversion of reflection seismic data, Geophysics, 48, 665- 676 https://doi.org/10.1190/1.1441497.

[10] Duffaut K., Alsos T., Landro, Rogno H., and Al-Najjar N. ( 200 ). Shear wave elastic impedance, The Leading Edge, 12221229.

[11] Inichinbia S., Sule P. O., Ahmed, A. L. and Hamza H., (2014). AVO inversion and lateral prediction of reservoir properties of Amangi hydrocarbon field of the Niger Delta area of Nigeria, IOSR Journal of Applied Geology and Geophysics, 2(2), pp. 8-17. https://doi.org/10.9790/0990-02220817.

[12] Inyang, Namdie J., Akpabio. O. and Agbasi Okechukwu E. (2017). Shale Volume and Permeability of the Miocene Unconsolidated Turbidite Sand of Bonga Oil Field, Niger Delta, Nigeria. Internationa Journal of Advanced Geosciences, 5 (1) $37-45$ https://doi.org/10.14419/ijag.v5i1.7586

[13] Inyang, N.J., Okwueze, E. E. and Agbasi, O. E. (2015), Detection of Gas Sands in the Niger Delta by Estimation of Poisson's DampeningFactor (PDF) Using Wireline Log Data, Geosciences, p-ISSN: 2163 1697, e-ISSN: 2163-1719, 2015; 5(1): 46-51.

[14] Keumsuk L., Dong-Geun Y., George M., Namsoon H., and Gwang H.L., (2013) A two-dimensional post stack seismic inversion for acoustic impedance of gas hydrate bearing deep-water sediments within the continental slope of the Ulleung Basin, East Sea, Korea, Terr. Atmos. Ocean. Sci., Vol. 24, No. 3, 295-310. https://doi.org/10.3319/TAO.2013.01.10.01(T).

[15] Okechukwu E. Agbasi, Magnus Igboekwe, Godwill Chukwu, Sunday E. Etuk (2018). Discrimination of Pore Fluid and Lithology of a Well in X Field, Niger Delta, Nigeria. Arabian Journal of Geoscience. 11(11) https://doi.org/10.1007/s12517-018-3610-7.

[16] Okoli Emeka Austin, Onyekuru Smauel I, Agbasi Okechukwu Ebuka and Zaidoon Taha Abdulrazzaq (2018). Application of Model-Based Inversion Technique in a Field in the Coastal Swamp Depobelt, Niger Delta. International Journal of Advanced Geosciences, 6 (1). 122 - 126. https://doi.org/10.14419/ijag.v5i2.7890.

[17] Orife J. M. and Avbovbo A.A., (2013). Stratigraphic and unconformity traps in the Nige Delta: AAPG Bulletine. 66(2): 1982, 251-262.
[18] Pendrel J., Seismic inversion, (2006). A critical tool in reservoir characterization, Scandinavian Oil-Gas Magazine, no. 5/6, pp. 1922.

[19] Russell B. H., Hedlin K., Hilterman F. J. and Lines L.R., Fluid-property discrimination with AVO: A Biot-Gassmann perspective, Geophysics, 68, 2003, 29-39. https://doi.org/10.1190/1.1543192.

[20] Ubong E. Essien, Akaninyene. O. Akankpo and Okechukwu. E. Agbasi (2017). Evaluation of Reservoir's Petrophysical Parameters, Niger Delta, Nigeria. International Journal of Advanced Geosciences, 5 (1) (2017) 19-25. https://doi.org/10.14419/ijag.v5i1.7456.

[21] Udo, K.I, Akpan, M. J., and Agbasi. O. E., (2015). Estimation of Overpressures in Onshore Niger Delta Using Wire-line Data. International Journal of Science and Research, Vol 4, Issue 5, pp. 2780 2784.

[22] Ushie F.A., Harry, T.A (2014): The Petrophysical Evaluation and Depositional Environment of Harrison 1 Well Using Core Data and Wireline Logs: Journal of Environment and Earth Science. www.iiste.orgISSN 2224-3216 (Paper) ISSN 2225-0948 (Online) Vol.4, No.9, pg. 125-133.

[23] Wagner C., Gonzalez A., Argawal V., Koesoemadinata A., Trares S. Biles N., and Fisger K., (2012). Quantitative application of post stack acoustic impedance inversion to subsalt reservoir development, The Leading Edge, 528-537. https://doi.org/10.1190/tle31050528.1.

[24] Whitcombe D. N., (20020. Elastic impedance normalization, Geophysics, v. 67, no. 1, p. 60-62. https://doi.org/10.1190/1.1451331. 